\title{
Increasing the traffic capacity of railway blocks
}

\author{
Boris Sergeev ${ }^{1, *}$, Vitaly Dontsov ${ }^{1}$, and Boris Arzhannikov ${ }^{1}$ \\ ${ }^{1}$ Ural State University of Railway Transport, 620034 Yekaterinburg, Russia
}

\begin{abstract}
The paper considers ways of increasing the traffic capacity of railway sections equipped with automatic block signaling systems, with a discrete variable number of block sections. Simultaneous traffic of long and short trains on a single block is analyzed. The paper highlights shortcomings and limitations of the existing automatic block signaling systems, including low traffic capacity and non-compliance with traffic safety requirements for railway sections. The study is aimed at removing these shortcomings and limitations. For this purpose, a reasonable technical solution has been presented, analyzed and scientifically grounded. System analysis techniques have been used to study the performance of traffic control devices.
\end{abstract}

\section{Introduction}

The key technical and economic indicator of railway sections is their traffic capacity, which is ensured by using various types of automatic block signaling systems with a discrete separation of the block length into several block sections. Issues related to improving the performance of such systems are studied by a number of Russian and international scientists and engineers [1-9]. Based on a scientific analysis, various technical solutions have been developed to improve the performance of automatic block signaling systems. In particular, a system that uses devices with a variable number of block sections is presented as one of optimal systems $[10,11]$. However, as shown by the study of well-known devices, they are not free of shortcomings. The purpose of this study is to create scientific foundations and develop general principles aimed at increasing the traffic capacity of railroad sections as related to trains of different lengths.

\section{Research methods}

The current methodology aimed at studying train traffic in railway sections is based on a system timing analysis of the processes that occur when trains move according to "proceed" or "stop" indications of the automatic block signaling system. In this case, the length of block sections on the Russian railways is determined in accordance with the applicable guidelines $[7,8]$, while the traffic capacity of the railway section can be calculated by the following formula [5]:

$$
N_{\text {block }}=1,440 / t_{\text {int }}
$$

\footnotetext{
*Corresponding author: sergeew@uralmail.com
} 
where: 1,440 is the number of minutes in a day, minutes; $t_{\text {int }}$ is the inter-train interval, minutes.

For the most common three-aspect automatic block signaling system, the minimum inter-train interval is defined as:

$$
t_{\text {int }}=0.06\left(3 L_{\mathrm{bs}}+l_{\mathrm{t}}\right) / V_{\mathrm{t}}
$$

where $3 L_{\mathrm{bs}}$ is the total length of block sections lying ahead of a moving train with length $l_{\mathrm{t}}$; $V_{\mathrm{t}}$ is the sectional speed of the train.

The length of each block section depends not only on the train speed, but also on a number of rated parameters of moving trains. This interaction of parameters can be presented in a generalized form as:

$$
L_{\mathrm{bs}}=k l_{\mathrm{p}}
$$

where $k$ is the coefficient that determines a set of parameters and is used in calculations performed in accordance with the standard [7]. These automatic block signaling systems are designed in such a way that any characteristics of trains moving along a designed block will meet the applicable traffic safety requirements as related to the braking distance and the maximum permissible speed. It is assumed that trains must move according to the green indication of the nearest traffic light lying ahead, i. e. at least three block sections $L_{\mathrm{bs}}$ ahead of a moving train must be clear. The number of reference cars (car with a standard length of $15 \mathrm{~m}$ ) moving along blocks ranges from 71 to 100 or 140 [8]. This range determines standard lengths of trains on different railway sections.

The calculated maximum length of a block section $L_{\mathrm{bs} \max }$ will be reached at the maximum train length $l_{\mathrm{t} \max }$ and respective values of the set of parameters $\Sigma \Pi_{i}$. This applies to freight trains, including long and heavy trains consisting of 100 or more reference cars. However, passenger or electric trains consisting of 6 to 26 reference cars move along the same railway section. Geometric, braking and traction characteristics of such trains $\left(\Sigma \Pi_{i}\right)$ are significantly different from those of freight trains [10].

Obviously, if practical lengths of block sections are determined by parameters of long trains, this will decrease the traffic capacity for passenger or electric trains.

Thus, there is a certain disproportion between possibilities of reaching a sufficiently high traffic for different types and lengths of rolling stock, which is determined by expressions (1) and (2).

Works $[10,11]$ propose a method to remove this disproportion. This method assumes that the number of block sections on the block varies with the length of the train that enters the block from a departure station. In other words, the number of signaling points switched on or off on the block varies with the train length. In order to implement this method at the departure station, it is necessary to determine the train length $l_{\mathrm{t}}$ using known axle counters on a track [6]. The collected information is transmitted to all signaling points of the block, some of which are switched off or on depending on $l_{\mathrm{t}}$ of the train leaving the departure station. In this case, inactive signaling points of the automatic block signaling system switch to the translation mode to translate the relevant codes through a rail circuit or channels of axle counters.

This method can be illustrated with a diagram of a block and a station with two trains whose lengths are $l_{\mathrm{t} 1}$ and $l_{\mathrm{t} 2}$ (Fig. 1). Information on the train length $S_{\mathrm{ax}}$ is transmitted from the axle counter to a signal box. It is converted into a signal $S$, which is sent to all signaling points of the block. According to this signal, the relevant signaling points of the automatic block signaling system are switched on or off. In the general case, as it clearly follows from the performed traction calculations based on the set of parameters $\Sigma \Pi_{i}$, the lengths of 
different block sections $l_{\mathrm{bs} \text { i }}$, according to the expression (3), will be different and close to the most efficient ones.

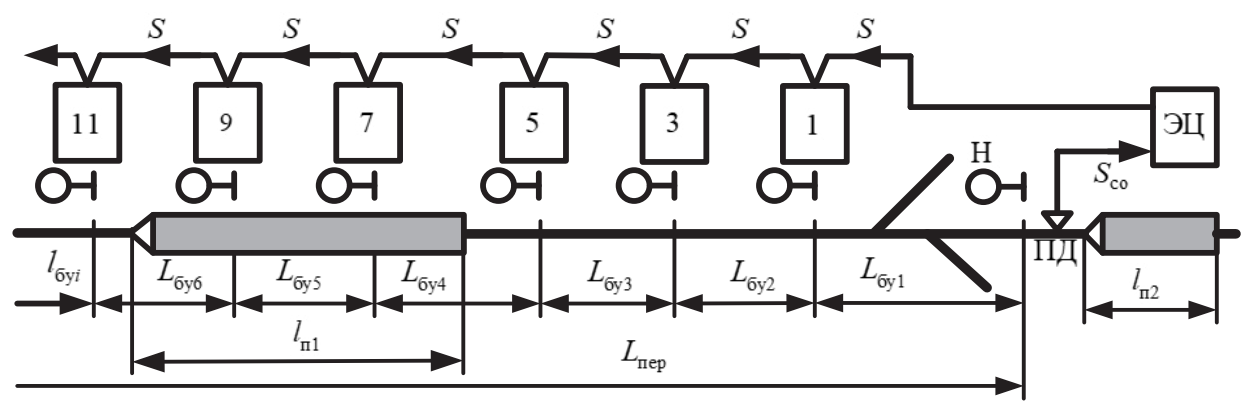

Fig. 1. Diagram of a block with simultaneous switching of all signaling points in the automatic block signaling system.

The practical application of this method can not only increase the traffic capacity of railway sections, but also optimize traffic control for heavy freight trains, in particular twin trains, based on signals of the automatic block signaling system. However, this approach to increasing the traffic capacity of blocks has some shortcomings. Let us illustrate them on the basis of the block diagram shown in Figure 1.

Suppose that the first long train with length $l_{\mathrm{t} 1}$ is moving along the block, covering the common length of several block sections: $l_{\mathrm{t} 1} \leq L_{\mathrm{bs} 6}+L_{\mathrm{bs} 5}+L_{\mathrm{bs} 4} ; l_{\mathrm{bs} 1}$. The second train leaving the station according to an exit signal $\mathrm{H}$ has a short length $l_{\mathrm{t} 2}$, which corresponds, e. g., to an electric train: $l_{\mathrm{t} 2} \leq l_{\mathrm{ts} \text { min }}$, where $l_{\text {ts min }}$ is the minimum length $i^{\text {th }}$ of the block section that lies, e. g., between signaling points 1 and 3 .

In this case, until the first train completely leaves the entire block $L_{\text {block}}$, all signaling points of the block will be in the initialization mode to initialize the long block sections whose total length in our case is equal to: $L_{\mathrm{ts} \Sigma}=L_{\mathrm{ts} 6}+L_{\mathrm{ts} 5}+L_{\mathrm{ts} 4}$.

Therefore, if several trains of different lengths are moving along the block and the first of them is a long train, then lengths of block sections for the second short train, which follows the first one, will correspond to lengths of the long train $l_{\mathrm{t} 1}$. Obviously, this restricts the speed of the second train along the block and its traffic capacity.

Another shortcoming of the existing method can be demonstrated on the same example with two trains moving along the block, the first of which is short, while the second one is long. In this case, when the first train with length $l_{\mathrm{t} \text { min }}$ leaves the station and enters the block, active / inactive signaling points will be configured in the form of various short block sections along the entire block. Obviously, the departure of the long train from the station will be impossible until the first train leaves the entire block. Only then, according to the signal $S$ (Fig. 1), signaling points of the block will be switched to a greater length $l_{\mathrm{t} 2}$ $>l_{\mathrm{t} 1}$. Failure to comply with this condition will not ensure the compliance with traffic safety requirements and, therefore, must be avoided.

Thus, this approach to increasing the traffic capacity can be optimized subject to the constraints arising from the fact that the approach is valid only in the presence and during movement of long trains along the block.

\section{Results}

The results of the scientific analysis indicated the possibility of eliminating these shortcomings. Let us consider this possibility using the example of the block in Figure 2, which shows the location and movement of two trains $l_{\mathrm{t} 1}$ and $l_{\mathrm{t} 2}$ of a specified length. The 
diagram in Figure 2 differs from the diagram in Figure 1 by the presence of information channels $S_{1 \mathrm{H}}, S_{3-1}, \ldots S_{11-9} \ldots$, which transmit data from signaling points of the block to adjacent signaling points and the signal box.

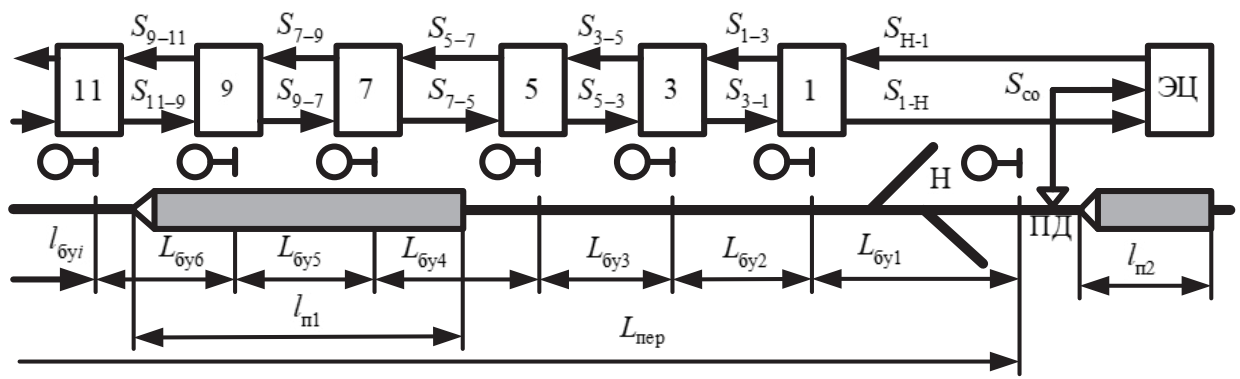

Fig. 2. Diagram of a block with selective switching of all signaling points in the automatic block signaling system.

The structural diagram of the block shown in Figure 2 works as follows. When the first, e. g., a long train of length $l_{\mathrm{t} 1}$ leaves the station and the block is clear according to signals $S_{\mathrm{H}-1}, S_{1-3}, \ldots S_{9-11}$, all signaling points receive the relevant information. This leads to initialization of only those signaling points that match the calculated set of parameters $\Sigma \Pi_{i}$ for this train.

When the train moves along the block and its tail end clears the relevant block sections, devices of signaling points generate the signals that are transmitted to adjacent signaling points and the signal box. Therefore, the station receives real-time information about leaving block sections by the tail end of the first train of length $l_{\mathrm{t} 1}$.

When the second train arrives at the station and is ready for departure, the axle counter transmits information about its length $l_{\mathrm{t} 2}$. This leads to the generation of signals $S_{\mathrm{H}-1}, S_{1-3}$, and $S_{3-5}$ (for the case shown on the block diagram in Figure 2). Then the departing second train will move along the block sections $L_{\mathrm{bs} 1}, L_{\mathrm{bs} 2}$ and $L_{\mathrm{bs} 3}$. As the first train moves to the signal box, information will come from signaling points $5,7,9,11$ and so on. This leads to change in indications of subsequent signals $S_{7-5}, S_{9-7}, S_{11-9}$ and so on. The system identifies a need to switch on or off certain signaling points, which are determined on the basis of traction calculations for another train length.

\section{Conclusion}

The study shows and justifies the possibility of changing the traffic capacity of a railway section at significantly different lengths of trains that move along the block. The schematic diagram of the proposed automatic block signaling system and its operation algorithm have been developed. By implementing the proposed traffic control algorithm for trains moving along the block, where signaling points and the signal box share information between each other through direct and reverse data transfer, it is possible to optimize traffic control processes for trains of arbitrary length and maximize the traffic capacity, while maintaining the required level of traffic safety.

At present, according to a planned schedule, specialists are preparing terms of reference for hardware and software implementation of the proposed automatic block signaling system using the accepted methodology for designing automation and remote control equipment in railway transport [12]. 


\section{References}

1. E. Masson, M. Barbinean, Wireless Communication for Railway Application (Springer, 2017).

2. The Railway Technical Website, Railway Technical, Available at: http://www.railway-technical.com/bookspapersarticles/railway-technical.com/ (February 15 2018).

3. Transport Canada, Rail Safety - FAQs, Available at: https://www.tc.gc.ca/eng/railsafety/railsafety-faq-969.html (February 1, 2018)

4. I.M. Kokurin, Proceedings of International Science and Practice Conference "Transport of Russia: Problems and Prospects", Vol. 2, 67-72 (Saint Petersburg, 2016). (In Russian)

5. P.A. Muginshtein, M.M. Mekhedov, Vestnik of the Railway Research Institute, Vol. 75(1), 3-11 (2016).

6. I.G. Tilk, Novye ustrojstva avtomatiki i telemehaniki zheleznodorozhnogo transporta [New automation and telemechanics devices of railway transport] (Ural State University of Railway Transport Publ., Yekaterinburg, 2010). (in Russian)

7. 660301. Regulations on automatic block signals location and defining lengths of block sections on the lines with automatic cab signaling (Saint Petersburg, 2003). (in Russian)

8. Optimization of transportation process optimization. Materials of The Board meeting of Science and Technology Council of the Ministry of Railway Transport of the RF, Zheleznodorozhnyj transport [Railway transport], 1, 2-4, (2001). (in Russian)

9. M.K. Jain, Average speed of freight train over Indian Railways, Available at: https:/www.railelectrica.com/status-of-train-speeds-over-indian-railways/averagespeed-of-freight-train-over-indian-railways/ (February 09, 2017).

10. I.G. Tilk, V.V. Lyanoy, M.A. Krivda, B.S. Sergeev, World of Transport and Transportation 4, 26-33, (2005). (in Russian)

11. I.G. Tilk, V.V. Lyanoy, M.A. Krivda, B.S. Sergeev, RU Patent 2288126 (2006). (in Russian)

12. V.V. Sapozhnikov, I.M. Kokurin, V.A. Kononov, Jekspluatacionnye osnovy avtomatiki $i$ telemehaniki [Operational foundations of automatics and telemechanics] (Marshrut, Moscow, 2006). (in Russian) 